\title{
Adolescent depression: Study protocol for a randomized, controlled, double-blind multicenter parallel group trial of Bright Light Therapy in a naturalistic inpatient setting (DeLight)
}

Martin Holtmann ${ }^{1 *}$, Laura Mokros ${ }^{1 \dagger}$, Inken Kirschbaum-Lesch ${ }^{1 \dagger}$, Michael Kölch ${ }^{2}$, Paul L. Plener ${ }^{3}$, Christian Ruckes ${ }^{4}$, Michael Schulte-Markwort ${ }^{5}$ and Tanja Legenbauer ${ }^{1}$

\begin{abstract}
Background: Depressive disorders are among the most prominent health problems in youth. Even with the best available pharmacological and non-pharmacological treatments, remission rates are low. Without early treatment, depression in youth is associated with a high risk of symptom progression, chronicity, co-morbidity, and suicidal behavior. Thus, adolescent depression is a prime candidate for innovation in treatment. In depressive adults, metaanalytic evidence has proven that bright light therapy (BLT) is a potent low-threshold intervention, promising due to easy application, low side effects, and optimized compliance. In adolescents, studies with small samples show promising effects. This randomized controlled trial will examine the effectiveness of BLT in youth inpatients.
\end{abstract}

Methods/design: In this randomized, controlled, double-blind multicenter parallel group trial, morning BLT is applied for four weeks in addition to treatment as usual (TAU) for depressed youth inpatients (daily morning exposure to bright light via light-emitting glasses, 10,000 Ix, for $30 \mathrm{~min}$ ) and will be compared to a control condition (placebo light treatment, red light, identical light glasses). The primary objective is to assess whether BLT reduces symptoms of depression in youth with greater effect compared to placebo light therapy. Secondary objectives are to examine the impact of BLT on responder status, application of antidepressant medication, and further depression-related symptoms (sleep, activity, quality of life, satisfaction with health, general psychopathology, alertness, and circadian function). $N=224$ patients will be recruited in a naturalistic inpatient setting. A follow-up will be carried out after three and six months.

Discussion: The study aims to discuss and evaluate BLT as an additive method supporting standardized clinical procedures dealing with severe to moderate depressive symptoms in youth.

Trial registration: German Clinical Trials Register, DRKS00013188. Registered on November 30, 2017.

Keywords: Depression in youth, Treatment, Bright light therapy, Inpatients

\footnotetext{
* Correspondence: martin.holtmann@lwl.org

† Laura Mokros and Inken Kirschbaum-Lesch contributed equally to this work.

${ }^{1}$ Clinic for Child and Adolescent Psychiatry, LWL University Hospital Hamm of the Ruhr-University Bochum, Heithofer Allee 64, 59071 Hamm, Germany

Full list of author information is available at the end of the article
}

(c) The Author(s). 2018 Open Access This article is distributed under the terms of the Creative Commons Attribution 4.0 International License (http://creativecommons.org/licenses/by/4.0/), which permits unrestricted use, distribution, and reproduction in any medium, provided you give appropriate credit to the original author(s) and the source, provide a link to the Creative Commons license, and indicate if changes were made. The Creative Commons Public Domain Dedication waiver (http://creativecommons.org/publicdomain/zero/1.0/) applies to the data made available in this article, unless otherwise stated. 


\section{Background}

Depressive disorders are among the most prominent health problems in youth. In adolescents, prevalence rates of depression are in the range of $5-6 \%$ [1], with a lifetime prevalence rate of $20 \%$ by late adolescence [2]. Prevalence in girls is twice as high as in boys and higher symptom load is found in youth with a high number of psychosocial risks [3]. The five-year recurrence rate in adolescent depression is as high as $40 \%$ [4]. In addition, a depressive mood during the winter season is quite common among youth in central Europe, with at least $2 \%$ of adolescents and young adults meeting criteria for seasonal affective disorder (SAD), e.g. a clinically meaningful depression with a seasonal pattern [5]. Of all depressive youth, $10 \%$ receive inpatient treatment at least once. In Germany, inpatient treatment rates for adolescent depression have increased dramatically by $>200 \%$ from 2000 to 2007, compared to $38 \%$ for all mental disorders [6].

Adolescent depression may interrupt educational attainment and thus affect human capital accumulation and future earnings. Since teenage suicide, for which depression is a principal risk factor, is the second leading cause of death in German youth, preceded only by traffic accidents [7], it is important to acknowledge that the human costs associated with depression are in a real sense greater than the economic costs. Adolescent depression is a key risk factor for adult depression; it severely impairs work and school management, leisure activities, and social contacts; frequency and degree of disabilities in depressed youth are at least as pronounced as in adults [8]. In short, depression in adolescents is prevalent, of significant public health importance, and thus a prime candidate for innovation in treatment.

Despite a huge increase in treatment research in the past 20 years, remission rates of pharmacological treatments are low [9]. In the carefully designed gold standard studies TADS and TORDIA, remission rates for moderate to severe depression were $<40 \%$ after three and six months of combined medication and psychotherapy $([10,11])$. A recent meta-analysis yielded only small effect-sizes for selective serotonin reuptake inhibitor (SSRI) and serotonin and norepinephrine reuptake inhibitor (SNRI) treatment of depressive disorders in children and adolescents [12]. Treatment decisions are further complicated by safety concerns of antidepressant-related self-harm and suicide in youth [13]. Despite these problems, a doubling of prescriptions for antidepressants in German adolescents has been observed in recent years [14]. The current evidence- and consensus-based clinical practice guideline of the German Association for Child and Adolescent Psychiatry (DGKJP) for the treatment of adolescent depression concluded that due to "the glaring lack of clinical studies (...) there is a pressing need for intervention research" [15].
Within the last decade, especially in the field of adult psychiatry, evidence suggests promising effects of bright light therapy (BLT) with respect to the improvement of depression symptoms. We conducted a systematic overview regarding BLT for the treatment of both non-seasonal and seasonal depression by using a broad range of databases. The present search is an update of already published reviews by our group laying special emphasis on clinical trials of BLT in depressed adolescents $([16,17])$. In adults, recent meta-analytic evidence [18] has proven that BLT leads to a significant reduction of depressive symptoms in non-seasonal depression (SMD $=-0.62, P<0.001)$. In particular, BLT appears to be efficacious when administered for $2-5$ weeks (SMD $=-0.78$, $P<0.001)$. The authors emphasize the obvious need to optimize the duration and intensity of exposure and the timing and duration of treatment sessions. In a Cochrane review on BLT in non-seasonal depression, studies with a higher methodological quality rating showed unequivocal superiority of BLT over control treatment (effect-size 0.90, 95\% confidence interval [CI] 0.31-1.50 [19]). Of note, treatment duration in almost all included studies was brief (1-2 weeks). Existing evidence suggests that longer treatment (at least four weeks) yields larger effects.

Up until now, only very few studies investigated the effects of BLT on depression in youth (see [20] for a systematic review of studies until 2012 and [18] for meta-analysis including more recent trials). In one of our pilot studies [21], remission rates after two weeks of BLT (light box, 10,000 lx; $n=30$ ) and a further three weeks of follow-up were $46.7 \%$ for BLT and $25.9 \%$ for dim light (100 lx; $n=27$ ); however, these differences were not significant due to the small sample size. BLT significantly improved sleep difficulties in youth [21]. Sleep restoration occurred only during BLT and remained stable during follow-up, while dim light remained without any effect. BLT may, therefore, be especially suited for depressive adolescents, of whom $75 \%$ are affected by problems with the sleep-wake rhythm [22]. A combination of BLT and an additional night with sleep restriction did not result in greater symptom reduction than BLT alone in 60 adolescent depressive inpatients [23]. A secondary analysis with actigraphy and sleep diary data showed greater sleep-enhancing effects in the BLT group than the combined group after two weeks of intervention [24].

BLT is promising due to robust evidence in adults, easy application, low side effects, and heightened compliance [25]. Light glasses have been shown to lead to the same results as a $10,000 \mathrm{~lx}$ light box $([26,27])$. However, empirical evidence on BLT for youth is scarce and pilot studies suffer from small sample sizes, lack of randomization, shortness of treatment duration, and lack of control settings. Moreover, a combination of BLT and treatment as usual (TAU; including pharmacotherapy) might be promising, because more naturalistic studies 
taking into account antidepressants do not exist. The proposed trial shall extend the pilot findings; treatment duration will be prolonged to four weeks to yield larger effects and follow-up will be extended to six months. Results of the proposed trial should allow conclusions as to the acceptability, the effects, and the costs of BLT in youth, thus providing a basis for decisions as to the broader implementation. We assume that BLT should lead to more robust symptom remission, potentially facilitating dose reduction or even replacement of antidepressants and a subsequent lower risk of adverse events (AEs). BLT may lead to a faster onset of effect [28], leading to shortened hospitalization, and can prevent transition to more severe forms of depression.

The projected trial will:

- examine whether BLT (daily morning exposure, $10,000 \mathrm{~lx}$, for $30 \mathrm{~min}$ ) in addition to multimodal TAU reduces symptoms of depression in youth inpatients with greater effect compared to placebo light therapy plus TAU (red light);

- assess the impact of BLT on responder status, application and dosage of antidepressant medication, and further depression-related symptoms (sleep, activity, quality of life, satisfaction with health, general psychopathology, alertness, circadian function), and length of stay in hospital;

- assess the prevalence and severity of BLT side effects which have been vastly neglected in previous studies.

\section{Methods/Design}

\section{Study rationale}

This trial is a randomized, controlled, double-blind multicenter parallel group study. The trial protocol has been approved by the medical ethics committee of the Ruhr-University Bochum, Germany (registration number 17-6140-BR) and is registered with the German Clinical Trials Register (DRKS00013188). The trial will be conducted according to the principles of ICH-GCP and appropriate legal regulations. The Professional Code for Physicians in Germany and Declaration of Helsinki in its actual version will be adhered to. For recommended items to address in a clinical trial protocol according to the SPIRIT 2013 Checklist, see "Additional file 1".

\section{Study setting and recruitment}

Four Departments of Child \& Adolescent Psychiatry of the Universities/Medical Schools of Bochum, Ulm, Hamburg, and Brandenburg/Neuruppin participate in the study and will recruit inpatients. The study sample should reflect everyday clinical practice to be as representative as possible for inpatient youth (aged 12-18 years) with moderate to severe depression [29]. Diagnoses will be ascertained by structured clinical interviews. To maximize generalizability (i.e. testing for effectiveness), exclusion criteria are limited to situations that would prevent full participation in the study or that might require additional treatment incompatible with study treatments. Inclusion and exclusion criteria are listed in Table 1. Antidepressant medication is allowed and controlled for in the analyses performed (naturalistic approach).

\section{Informed consent process}

First, patients and parents will be informed in written and oral forms regarding the study, the procedures, and the potential risks or discomforts and potential benefits. An informed consent document with details about the study including its purpose, duration, procedures, and key contacts, as well as risks and potential benefits, will be handed out to patients and parents. The patients and parents will then decide whether to give written consent. Patients or parents unwilling or unable to consent will not be included in the study. At any time, participants or parents may withdraw their consent for any reason without any negative consequences regarding their treatment.

\section{Randomization and blinding procedures}

Potential bias is minimized by randomized treatment allocation and by blinded outcome assessment. Since patients will be blinded towards their treatment, patient-reported outcomes including the primary outcome will be blinded. Independent raters unaware of the patient's treatment will perform further assessments (CDRS-R). Randomization is performed using the electronic case report form and will be stratified by prior pharmacological antidepressant therapy (yes/no), trial site, and sex. The randomization ratio will be 1:1 based on blocks of variable length.

Patients and clinical raters will both be blinded regarding treatment allocation. While the primary outcome (BDI-II) will be rated by the blinded patients themselves, the clinical evaluation (CGI-I, CDRS-R) will be performed by the blinded clinician. Blinding will be assured by separating diagnostic procedures from general patient

\begin{tabular}{|c|c|}
\hline \multirow{3}{*}{$\begin{array}{l}\text { Inclusion } \\
\text { criteria }\end{array}$} & Moderate to severe depression (BDI-II) \\
\hline & Inpatients aged $12-18$ years \\
\hline & $\begin{array}{l}\text { Written informed consent of the inpatient and } \\
\text { the caretaker }\end{array}$ \\
\hline \multirow{6}{*}{$\begin{array}{l}\text { Exclusion } \\
\text { criteria }\end{array}$} & Acute suicidality, bipolar 1 disorder, or schizophrenia \\
\hline & Pregnancy or lactation \\
\hline & $\begin{array}{l}\text { Treatment with beta-blocker or with high-potency } \\
\text { neuroleptics }\end{array}$ \\
\hline & Diseases of the retina \\
\hline & $\mathrm{IQ}<70$ \\
\hline & Non-German-speaking child or caretaker \\
\hline
\end{tabular}

BDI-II Beck Depression Inventory II 
and data handling. There will be one research assistant organizing recruitment and all patient-relevant data handling as well as a second person solely performing the diagnostic assessments. This procedure has proven to be feasible in our pilot study [21].

\section{Experimental / control condition}

Finding an appropriate control condition for light therapy to minimize placebo effects is difficult, since these effects are expected to be quite high in depression. In the projected study, the active light treatment will consist of daily morning exposure to white fluorescent light (light glasses Luminette; $10,000 \mathrm{~lx}$ ) for $30 \mathrm{~min}$ for 28 days (weekends excluded), while the placebo treatment will be provided by identical glasses with a red light without known effects on depression. This design has been shown to be feasible in adolescents [21]. Since light therapy is most effective if applied at an optimal time in the circadian phase of the patient, light interventions will be carried out 7.5-9.5 $\mathrm{h}$ after the individual evening melatonin onset (DLMO) estimated by the MorningnessEveningness Questionnaire (MEQ) [30, 31].

\section{Interventions}

Following feedback from the participants in our pilot studies, light boxes are replaced by light glasses that allow patients to carry on with everyday tasks and fit into their daily routine while giving the same results as a $10,000 \mathrm{~lx}$ light box $([26,27])$. To ensure an appropriate and constant number of lux administered in each condition, intensities are quantified using a Lux meter (PCE-172). If a patient is not able to receive the intervention on a given day (e.g. due to illness), he is committed to prolong the intervention phase for one day. In case of several missing interventions (max. 20\% of the sessions), the patient will be excluded from the study. In order to control for placebo effects, the therapy expectancy and credibility questionnaire will be administered in a modified version before treatment.

Study interventions will be added to a naturalistic multimodal inpatient TAU. TAU will be documented in detail: due to the requirements of the recently introduced new reimbursement system for psychiatric hospitals in Germany, the participating centers are already documenting continuously type, frequency, and duration of patient-directed interventions electronically for all relevant professions (psychiatrists, psychotherapists, nurses, occupational therapists, etc.). These data can be aggregated for participants of the projected study to determine the distribution among groups.

In some cases, psychopharmacological antidepressant treatment may be warranted and will be provided according to the attending physician's clinical judgment (non-interventional). Afternoon outdoor activities are part of a comprehensive youth inpatient care but are unlikely to interfere with the antidepressant response to BLT, since treatment response is maximized if BLT is initiated no later than $8.5 \mathrm{~h}$ after a patient's estimated melatonin onset, e.g. only in the early morning. Light exposure at midday or in the afternoon does not result in an antidepressant effect [32].

\section{Intervention scheme / trial flow}

Inpatients from all trial sites that meet the inclusion criteria and give informed consent are consecutively included into the trial within one week after admission. After inclusion, inpatients will be interviewed by the blinded research psychologist. Initial self-report measurements and objective data in relation to depression and associated co-morbidity as well as medication are assessed by a trained diagnostician. In addition, treatment expectancy and responsibility to seasonal effects will be assessed. After the pre-treatment assessment (T1), patients are randomized to either a placebo control condition (PCC) or BLT. Both groups also receive TAU. Trial interventions will be delivered each morning (except weekends) over a period of four weeks about $7.5 \mathrm{~h}$ after the DLMO assessed with the MEQ.

During the intervention trial, an interim assessment with the BDI-II after two weeks will take place in order to control patient-perceived changes in depression more closely. There is no full assessment in order to reduce stress for the patients. Four weeks after completion of the treatment, individuals will again be interviewed by the blinded research psychologist and score the self-rating questionnaires as well as perform the neuropsychological assessment and deliver saliva melatonin and cortisol (post-treatment assessment; T2). Three and six months after T2, follow-up assessments will be performed via telephone and questionnaires to control for stability (T3 and T4). There is no full reassessment for neurobiological and neuropsychological data in order to facilitate the response to fulfil the follow-up assessment and to meet patients' needs best. Moreover, AEs will be assessed in both treatment groups on a weekly basis. For an overview of the trial flow, see Fig. 1. For details on procedures per time point, see Figs. 2 and 3.

\section{Measurements}

The primary endpoint of the study is the change in patient-perceived severity of depressive symptoms after treatment, assessed with the BDI-II.

\section{Beck Depression Inventory-II}

The BDI-II is a self-rating instrument that is extensively used in clinical studies worldwide to assess the degree of depressive symptoms of the last seven days (validated in German adolescents; [33]). The score is easily calculated 


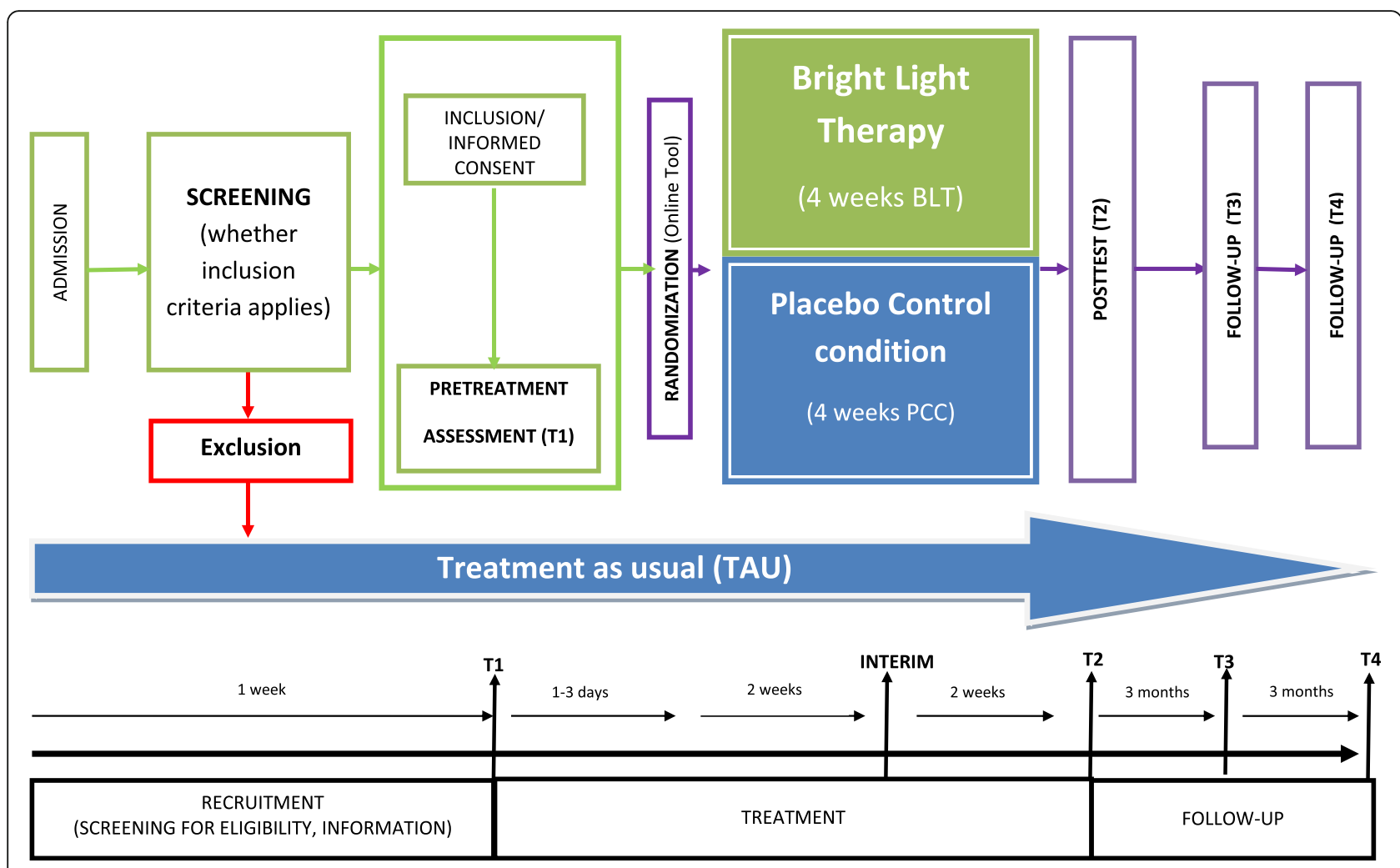

Fig. 1 Trial flow overview. PCC placebo control condition + TAU, BLT Bright Light Therapy + TAU; T1 assessment before treatment, T2 assessment at the end of the four-week treatment, T3 follow-up after three months

as a sum score. This score will be used in a dimensional way in the analysis, as this provides more power than a categorical use. According to NICE guidelines, the clinically relevant difference between treatment groups is 3 BDI-II scale points. The BDI-II standard deviation is expected to be about 10 scale points. The study aims to show the same treatment differences as in our pilot study [21] of about 4.37 scale points, which is clearly a relevant treatment difference.

The secondary endpoints include independent evaluator-, adolescent-, parent-, and clinician-rated measures of depression, improvement and impairment, and functional status. In addition, measures are included to allow the exploratory examination of a focused set of putative moderators and mediators of treatment response, including demographics, treatment and medical history, diagnostic-level data for depression and co-morbid conditions, scalar measures of internalizing and externalizing symptoms, family functioning and environment, peer relations, academic and school performance, quality of life, and treatment-related expectancies and beliefs:

\section{Clinical Global Impression (CGI)}

Blinded assessment of the global severity of the symptoms (CGI-S) at baseline and global change in degree of illness compared to the baseline dichotomized at much improved or better on the Clinical Global Impressions-Improvement (CGI-I) scale [34] rated by an experienced blinded clinical investigator.

\section{Children's Depression Rating Scale-Revised (CDRS-R)}

Clinician-rated reduction of depressive symptoms, assessed by a blinded investigator after four weeks of treatment (at T2). The CDRS-R $([35,36])$ is a widely used semi-structured interview to assess clinician-rated depressive symptoms. It has been applied in various multicenter studies to assess efficiency of pharmacotherapy and psychotherapy in adolescents (e.g. TADS, [37]; ADAPT, [38]). It shows good psychometric properties $($ Cronbach's Alpha $=0.85$; inter-rater reliability $=0.92)$.

\section{Antidepressant medication status}

Changes in the treatment regime (initiation/cessation/ dose-adjustment of antidepressants) will be assessed weekly from baseline to T2 and in the follow-ups.

\section{Sleep quality and effectiveness}

Change in sleep quality is assessed via the self-report Sleep-Questionnaire B (SFB/R; [39]), a well validated measure sensitive to treatment changes. 


\begin{tabular}{|c|c|c|c|c|c|c|c|c|}
\hline & \multicolumn{8}{|c|}{ STUDY PERIOD } \\
\hline & \multirow{2}{*}{$\begin{array}{c}\text { Enrolment } \\
0\end{array}$} & \multirow{2}{*}{$\begin{array}{c}\text { Allocation } \\
0\end{array}$} & \multicolumn{5}{|c|}{ Post-allocation } & \multirow[t]{2}{*}{ Close-out } \\
\hline${\text { TIMEPOINT }{ }^{\star \star}}$ & & & $t_{1}$ & Interim & $t_{2}$ & $t_{3}$ & $t_{4}$ & \\
\hline Trial days & -7 & -2 & 1 & 14 & 28 & 120 & 210 & \\
\hline \multicolumn{9}{|l|}{ ENROLMENT: } \\
\hline $\begin{array}{r}\text { Diagnosis } \\
\text { depressive } \\
\text { disorder }\end{array}$ & $\mathrm{x}$ & & & & & & & \\
\hline Eligibility screen & $\mathrm{x}$ & & & & & & & \\
\hline Informed consent & $\mathrm{x}$ & & & & & & & \\
\hline Randomization & & $\mathrm{x}$ & & & & & & \\
\hline \multicolumn{9}{|l|}{ INTERVENTIONS } \\
\hline \multicolumn{9}{|l|}{$\begin{array}{c}\text { Bright Light } \\
\text { Therapy }\end{array}$} \\
\hline \multicolumn{9}{|l|}{$\begin{array}{r}\text { Placebo Control } \\
\text { Condition }\end{array}$} \\
\hline \multicolumn{9}{|l|}{ ASSESSMENTS: } \\
\hline BDI-II & $\mathrm{x}$ & & & $x$ & $x$ & $x$ & $\mathrm{x}$ & \\
\hline CDRS-R & $\mathrm{x}$ & & & & $x$ & $x$ & $\mathrm{x}$ & \\
\hline 10 & $\mathrm{x}$ & & & & & & & \\
\hline \multirow{3}{*}{$\begin{array}{l}\text { Demographics } \\
\text { (e.g. sex, age) } \\
\text { Previous and } \\
\text { concomitant } \\
\text { medication } \\
\text { Previous and } \\
\text { concomitant } \\
\text { diseases }\end{array}$} & $x$ & & & & & & & \\
\hline & & & $x$ & & & & & \\
\hline & & & $x$ & & & & & \\
\hline \multirow[t]{2}{*}{ CGI-S } & & & $x$ & & & & & \\
\hline & & & & & $\mathrm{x}$ & $\mathrm{x}$ & $\mathrm{x}$ & \\
\hline CGI-I & & & $\mathrm{x}$ & & & & & \\
\hline \multirow{2}{*}{$\begin{array}{c}\text { SF-BR } \\
\text { KIDSCREEN10 }\end{array}$} & & & $\mathrm{x}$ & & $x$ & $\mathrm{x}$ & $\mathrm{x}$ & \\
\hline & & & $x$ & & $x$ & $x$ & $x$ & \\
\hline CHIP-AE & & & $x$ & & $x$ & $x$ & $\mathrm{x}$ & \\
\hline SDQ & & & $\mathrm{x}$ & & $\mathrm{x}$ & $\mathrm{x}$ & $\mathrm{x}$ & \\
\hline TAPb & & & $\mathrm{x}$ & & $\mathrm{x}$ & & & \\
\hline \multirow[t]{2}{*}{ Actigraphy } & & & $x$ & & $\mathrm{x}$ & & & \\
\hline & & & $\mathrm{x}$ & & $x$ & & & \\
\hline Saliva Melatonin & & & $\mathrm{x}$ & & $\mathrm{x}$ & & & \\
\hline \multirow{2}{*}{$\begin{array}{l}\text { Saliva Cortisol } \\
\text { Antidepressant } \\
\text { medication } \\
\text { status } \\
\text { Treatment } \\
\text { Expectancy }\end{array}$} & & & $x$ & $x$ & $\mathrm{x}$ & $x$ & $x$ & \\
\hline & & & $x$ & & & & & \\
\hline \multirow{2}{*}{$\begin{array}{l}\text { Adverse Events } \\
\text { Analysis of } \\
\text { Study Outcomes }\end{array}$} & & & & $x$ & $x$ & & & \\
\hline & & & & & & & & $\mathrm{x}$ \\
\hline
\end{tabular}

Fig. 2 (See legend on next page.) 
(See figure on previous page.)

Fig. 2 Study process schedule (according to SPIRIT guidelines). T1 pre-treatment assessment, T2 post-treatment assessment, T3 follow-up three months after T2, T4 follow-up six months after T2, BDI-II Beck Depression Inventory II, CDRS-R Children's Depression Rating Scale-Revised, CGI-S Clinical Global Impression-Severity, CGI-I Clinical Global Impression-Improvement, SPAQ Seasonal Pattern Assessment Questionnaire, SFBR Sleep Questionnaire-B/Revised, KIDSCREEN10 Health Related Quality of Life self-rating assessment - short version, CHIP-AE Child Health and IIIness Profile-Adolescent Edition, SDQ Strength and Difficulties Questionnaire, TAP Testbattery for Attentional Performance; ${ }^{a}$ only subdomain physical activity administered; ${ }^{b}$ only Alerteness subtest administered

\begin{tabular}{|c|c|c|c|c|c|}
\hline Action & $\begin{array}{l}\text { Screening/ } \\
\text { Recruitment }\end{array}$ & $\begin{array}{l}\text { Pretreatment } \\
\text { (T1) }\end{array}$ & Interim & $\begin{array}{l}\text { Posttreatment } \\
\text { (T2) }\end{array}$ & $\begin{array}{l}\text { Follow-up } \\
\text { (T3 and T4) }\end{array}$ \\
\hline Trial days & -7 & 1 & 14 & 28 & 112 and 168 \\
\hline $\begin{array}{l}\text { Diagnosis depressive } \\
\text { disorder }\end{array}$ & $x$ & & & & \\
\hline Inclusion/exclusion criteria & $x$ & & & & \\
\hline $\begin{array}{l}\text { Patient information and } \\
\text { consent }\end{array}$ & $x$ & & & & \\
\hline $\begin{array}{l}\text { Demographics (e.g. sex, } \\
\text { age) }\end{array}$ & & $x$ & & & \\
\hline $\begin{array}{l}\text { Previous and concomitant } \\
\text { medication }\end{array}$ & & $x$ & & & \\
\hline $\begin{array}{l}\text { Previous and concomitant } \\
\text { diseases }\end{array}$ & & $x$ & & & \\
\hline Physical examination & & $x$ & & & \\
\hline Randomisation & $x$ & & & & \\
\hline $\begin{array}{l}\text { BDI-II (primary outcome } \\
\text { score) }\end{array}$ & & $x$ & $x$ & $x$ & $x$ \\
\hline CGI-status & & & & $\mathrm{x}$ & $x$ \\
\hline CDRS-R & & $x$ & & $\mathrm{x}$ & $x$ \\
\hline SF-B/R & & $x$ & & $x$ & $x$ \\
\hline SDQ & & $\mathrm{x}$ & & $\mathrm{x}$ & $x$ \\
\hline KIDSCREEN10 & & $x$ & & $x$ & $x$ \\
\hline CHIP-AE & & $x$ & & $x$ & $x$ \\
\hline Actigraphy & & $x$ & & $x$ & \\
\hline TAPb & & $x$ & & $x$ & \\
\hline Saliva Melatonin & & $x$ & & $x$ & \\
\hline Saliva Cortisol & & $x$ & & $x$ & \\
\hline MEQ & & $x$ & & $x$ & $x$ \\
\hline SPAQ & & $x$ & & & \\
\hline Treatment Expectancy & & $x$ & & & \\
\hline Adverse Events & & & $\mathrm{x}$ & $\mathrm{x}$ & $x$ \\
\hline
\end{tabular}

Fig. 3 Overview of time-points of visits and procedures per time-point 


\section{Level of activity}

Assessed in an objective, ecological valid way via actigraphy (a watch measuring movement and light exposure before/after treatment, assessing time in bed and activity); ecological validity has been proven empirically (e.g. [40]). State-of-the-art software and algorithms will be applied to derive the parameters of interest (e.g. changes in the total degree of activity, time in bed, and sleep efficiency).

\section{Health-related quality of life (HRQoL)}

Changes in an individual's perception of their wellbeing are assessed with the KIDSCREEN-10, a short, valid, and reliable self-report, providing a total score reflection of HRQoL [41].

\section{Physical activity}

The Child Health and Illness Profile-Adolescent Edition (CHIP-AE) is a generic measure of satisfaction with health providing several domains and subdomains. The CHIP-AE is sensitive to treatment effects in adolescents [42]. Here, the domain Resilience with the subdomain Physical Activity shall be assessed, providing information about involvement in a variety of activities related to fitness [43].

\section{General psychopathology}

Changes in general psychopathology are assessed via self and parent versions of the Strength and Difficulties Questionnaire (SDQ). Its change sensitivity allows estimating treatment efficacy on affective/behavioral symptoms. The validated German version [44] has, for example, been used in the KiGGS survey.

\section{Alertness}

BLT has direct effects on alertness. Changes in level of alertness will be assessed via the subtest "Alertness" of the Testbattery for Attentional Performance (TAP) [45].

\section{Neurobiological indices of circadian function}

Two "gold standard" indices of circadian function will be assessed: the DLMOand the cortisol awakening response (CAR). Changes in DLMO from before to after treatment will indicate direct impact of BLT on circadian functioning, while a low pre-treatment CAR has been reported to predict the response to BLT in adults with non-seasonal depression [46].

\section{IQ}

The German "Zahlen-Verbindungs-Test" (ZVT) [47] is used to assess the intelligence quotient. The ZVT is a trail-making and speed test in which participants are instructed to connect numbers from 1 to 90 which are randomly positioned on a piece of paper. It shows sufficient psychometric properties (test reliability $=0.81-0.97$ ) [48].

\section{Seasonal pattern}

To control for seasonal effects, the Seasonal Pattern Assessment Questionnaire (SPAQ) will be administered to calculate a total "seasonality score" [49].

\section{Data analysis \\ Proposed sample size/power calculations}

In our pilot study [20], a similar trial design was used, investigating the effects of BLT versus placebo light therapy after a treatment period of 14 days and a follow-up period of three weeks. Remission rates at the end of follow-up (week 5) were $46.7 \%$ for BLT and $25.9 \%$ for dim light. These remission rates were transformed ( $2 *$ arcsin; $\sqrt{ }$ remission rate). The difference between the transformed remission rates resulted in Cohens $h$ of 0.437 and was used for the calculation of the study sample. A significant difference between the two groups is defined as 3 points on the BDI-II by the NICE guidelines. A standard deviation of 10 points in the BDI-II is assumed. Thus, the proposed study aims to show a group difference of 4.37 points on the BDI-II. Calculations based on this difference yield a sample size of 224 patients (112 for each group) when using an independent $\mathrm{t}$-test at a significance level of $5 \%$ and a power of 90\%. Sample size was calculated using PROC POWER in $\mathrm{SAS}^{\circ}$ Version 9.4. Drop-outs will be analyzed using multiple imputation methods.

\section{Statistical analyses}

The primary analysis population is the intention-to-treat (ITT) population comprising all randomized patients. Group difference in change of BDI-II rating under trial treatment will be analyzed by an analysis of covariance (ANCOVA) comprising the covariates baseline BDI-II rating, treatment group, prior pharmacological antidepressant therapy (yes/no), and sex. Missing values will be replaced by multiple imputation. The two-sided level of significance will be $\alpha=5 \%$. Supportive analyses are planned for the Per Protocol Population and the Completer Population. The Per Protocol Population comprises all patients from the ITT population without major protocol violations: lack of compliance with regard to therapy sessions; not meeting all inclusion criteria; meeting at least one exclusion criterion; intake of unallowed concomitant medication during the study; end of treatment visit out of schedule. In an additional sensitivity analysis, missing values will be analyzed based on the last available BDI-II rating (Last Observation Carried Forward [LOCF]). In case the LOCF approach turns out to be not conservative, an additional Baseline Observation Carried Forward (BOCF) analysis will be performed.

Further subgroup analyses are planned to detect possible differences between predictive factors (e.g. trial site, concomitant psychotropic medication for co-morbid disorders, 
baseline CAR and DLMO, type of depression [seasonal/ non-seasonal], season, expectancy questionnaire). No interim analyses are planned. Secondary endpoints will be analyzed descriptively applying appropriate methods depending on the scale of the respective parameter. Interpretation of statistical tests must be exploratory except for the primary outcome.

AEs will be coded according to MedDRA terminology. Detailed information collected for each AE will include: a description of the event; duration; whether the $\mathrm{AE}$ was serious; intensity; relationship to trial treatment; action taken; and clinical outcome. Summary tables will present the number of participants observed with AEs by MedDRA System Organ Class and Preferred Term and corresponding percentages. Additional subcategories will be based on event intensity and relationship to trial treatment. A subject listing of all AEs will be prepared.

\section{Discussion}

In this study protocol, we presented the background, design, measurements, and statistical procedures of our randomized, controlled, double-blind multicenter parallel group trial of BLT in a naturalistic inpatient setting. We discussed the medical problems regarding treatment and remission rates in adolescents meeting the criteria of depression and constituted the need to close the gap of evidence of BLT in youth therapy by prolonged (four weeks) intervention time and minimized bias problems due to our randomized, double-blind design.

There are several limitations the study will have to deal with, e.g. the exclusion of the weekends during intervention periods or lack of evidence for actual wearing time of the glasses because of the naturalistic setting. An additional disadvantage is the already regulated sleep-wake rhythm due to the inpatient setting, making a larger difference between activity- and sleep-times among the patients of the two treatment groups difficult to emerge. Moreover, the strict schedules at the infirmaries limit the power of the results compared to a more realistic, ambulant setting.

As remission rates of state-of-the-art treatments for depressed adolescents such as cognitive behavioral therapy and antidepressants remain unsatisfactory, and due to its easy application, low side effects, and optimized compliance, BLT seems to be a promising add-on therapy to psychological and pharmacological approaches to increase standard treatment effects. Since previous studies investigating the effect of BLT in depressed adolescents suffer from small sample sizes, the results of this projected trial will be of great importance.

\section{Trial status}

Recruitment is planned to begin in February 2018.

\section{Additional file}

Additional file 1: SPIRIT 2013 Checklist: Recommended items to address in a clinical trial protocol and related documents*. (DOCX $54 \mathrm{~kb}$ )

\section{Abbreviations}

ANCOVA: Analysis of covariance; BDI-II: Beck Depression Inventory II; BLT: Bright light therapy; BOCF: Baseline Observation Carried Forward; CAR: Cortisol awakening response; CDRS - R: Children's Depression Rating Scale - Revised; CGI-I: Clinical Global Impression-Improvement; CGI-S: Clinical Global Impression-Severity; CHIP - AE: Child Health and Illness ProfileAdolescent Edition; DALYs: Disability Adjusted Life Years; DGKIP: German Association for Child and Adolescent Psychiatry; DLMO: Dim light melatonin onset; HRQoL: Health-related quality of life; ICH-GCP: International Council for Harmonization of Technical Requirements for Pharmaceuticals for Human Use (ICH) - Good Clinical Practice; ITT: Intention-to-treat;

IZKS: Interdisziplinäres Zentrums Klinische Studien; KIGGS: Studie zur Gesundheit von Kindern und Jugendlichen in Deutschland; LOCF: Last Observation Carried Forward; MedDRA: Medical Dictionary for Regulatory Activities; MEQ: Morningness-Eveningness Questionnaire; NICE: National Institute for Health and Care Excellence; PCC: Placebo control condition; SAD: Seasonal affective disorder; SAE: Serious adverse events; SDQ: Strengths and Difficulties Questionnaire; SF-B/R: Schlaffragebogen B - Revidierte Fassung; SPAQ: Seasonal Pattern Assessment Questionnaire; T1: Pre-treatment assessment; T2: Post-treatment assessment after four weeks; T3: Post-treatment assessment after three months; T4: Post-treatment assessment after six months; TADS: Treatment of adolescents with depression study; TAP: Testbatterie zur Aufmerksamkeitsprüfung; TAU: Treatment as usual; TORDIA: Treatment of resistant depression in adolescents; UKE: Universitätsklinikum Hamburg-Eppendorf

\section{Acknowledgements}

We gratefully acknowledge financial support from the German Ministry of Education and Research (Bundesministerium für Bildung und Forschung, BMBF).

\section{Funding}

The study is funded by the German Ministry of Education and Research (01KG1713).

Availability of data and materials

Data and materials are available from the corresponding author upon request.

\section{Authors' contributions}

$\mathrm{MH}$ and $\mathrm{TL}$ conceived the research project; $\mathrm{MH}$, TL, and CR designed the study; and $M H, T L, C R, L M$, and IK designed and tailored the study protocol. MK, PP, and MS helped to draft the manuscript. All authors read and approved the final manuscript.

\section{Ethics approval and consent to participate}

The study has been approved by the medical ethics committee of the RuhrUniversity Bochum, Germany (registration number 17-6140-BR). The trial will be conducted according to the principles of ICH-GCP and appropriate legal regulations. Professional Code for Physicians in Germany and Declaration of Helsinki in its actual version will be adhered to. All participants and their parents will need to sign an informed consent document with details about the study including its purpose, duration, procedures, and key contacts, as well as risks and potential benefits. At any time, participants or parents may withdraw their consent for any reason without any negative consequences regarding their treatment.

\section{Consent for publication}

Not applicable.

\section{Competing interests}

Prof. Holtmann served in an advisory role for Shire and Medice and received conference attendance support or was paid for public speaking by Lilly, Medice, Neuroconn, and Shire. He receives research support from the German Research Foundation and the German Ministry of Education and Research. He receives royalties as editor in chief of the German Journal for Child and Adolescent Psychiatry and for text books from Hogrefe. Prof. Legenbauer received conference attendance support from Lilly as well as royalties for text books from Hogrefe and Springer as well as research support from the German Ministry of Education and Research, the European Union (Department of Justice), the Swiss anorexia nervosa foundation (SANS) 
and the FORUM Institute of the Ruhr-University Bochum. Prof. Koelch received research support from the German Ministry of Education and Research, German Ministry of Health, German Ministry for Families, Women, Seniors and Youth, Swiss Office for Justice; he receives royalties for books or chapters in books by Hogrefe, Springer, and Beltz. Prof. Plener received speaker honorarium from Shire and royalties from text books by Hogrefe and Springer. Within the last five years he received research support from The German Federal Ministry of Education and Research (BMBF), the German Federal Institute for Drugs and Medical Devices (BfArM), the WW Foundation, the Baden-Wuerttemberg Foundation, the Foundation for outpatient child and adolescent psychiatry, Servier, and Lundbeck. Prof. Schulte-Markwort received research support of BMBF, innovation foundation, and conference attendance support by Medice.

The present work is unrelated to the above grants and relationships. The other authors declare that they have no competing interests.

\section{Publisher's Note}

Springer Nature remains neutral with regard to jurisdictional claims in published maps and institutional affiliations.

\section{Author details}

${ }^{1}$ Clinic for Child and Adolescent Psychiatry, LWL University Hospital Hamm of the Ruhr-University Bochum, Heithofer Allee 64, 59071 Hamm, Germany. 2Department Child and Adolescent Psychiatry and Psychotherapy, Brandenburg Medical School, Neuruppin, Germany. ${ }^{3}$ Department of Child and Adolescent Psychiatry and Psychotherapy, Ulm University, UIm, Germany. ${ }^{4}$ Interdisciplinary Centre for Clinical Trials, Mainz University, Mainz, Germany. ${ }^{5}$ Department Child and Adolescent Psychiatry, Hamburg University, Hamburg, Germany.

Received: 30 November 2017 Accepted: 28 September 2018

\section{Published online: 19 October 2018}

\section{References}

1. Ravens-Sieberer U, Wille N, Erhart M, Bettge S, Wittchen H-U, Rothenberger A, et al. Prevalence of mental health problems among children and adolescents in Germany: results of the BELLA study within the National Health Interview and Examination Survey. Eur Child Adolesc Psychiatry. 2008;17(1):22-33.

2. Birmaher B, Brent D, Bernet W, Bukstein O, Walter H, Benson RS, et al. Practice parameter for the assessment and treatment of children and adolescents with depressive disorders. J Am Acad Child Adolesc Psychiatry. 2007;46(11):1503-26

3. Bettge S, Wille N, Barkmann C, Schulte-Markwort M, Ravens-Sieberer U. BELLA study Group. Depressive symptoms of children and adolescents in a German representative sample. Eur Child Adolesc Psychiatry. 2008;7(1):71-81.

4. Harrington R, Fudge $H$, Rutter M, Pickles A, Hill J. Adult outcomes of childhood and adolescent depression. Arch Gen Psychiatry. 1990;47:465-73.

5. Steinhausen HC, Gundelfinger R, Winkler Metzke C. Prevalence of selfreported seasonal affective disorders and the validity of the seasonal pattern assessment questionnaire in young adults. Findings from a Swiss community study. J Affect Disord. 2009;115:347-54

6. Holtmann M, Duketis E, Poustka L, Zepf FD, Poustka F, Bölte S. Bipolar disorder in children and adolescents in Germany: National trends in the rates of inpatients, 2000-2007. Bipolar Disorder. 2010;12:155-63.

7. Becker K, Adam H, In-Albon T, Kaess M, Kapusta N, Plener PL. Assessment and therapy of suicidality in adolescence: the most important recommendations of the current guideline. Z Kinder Jugendpsychiatr Psychother. 2017:45:485-97. https://doi.org/10.1024/1422-4917/a000516.

8. Wittchen HU, Jacobi F, Rehm J, Gustavsson A, Svensson M, Jönsson B, et al. The size and burden of mental disorders and other disorders of the brain in Europe 2010. Eur Neuropsychopharmacol. 2011;21:655-79.

9. Emslie GJ, Kennard BD, Mayes TL, Nakonezny PA, Zhu L, Tao R, et al. Insomnia moderates outcome of serotonin-selective reuptake inhibitor treatment in depressed youth. J Child Adolesc Psychopharmacol. 2012;22(1):21-8.

10. Kennard B, Silva S, Vitiello B, Curry J, Kratochvil C, Simons A, et al. Remission and residual symptoms after short term treatment in the treatment of adolescents with depression study (TADS). J Am Acad Child Adolesc Psychiatry. 2006;45:1404-11.
11. Emslie GJ, Mayes T, Porta G, Vitiello B, Clarke G, Wagner KD, et al. Treatment of resistant depression in adolescents (TORDIA): week 24 outcomes. Am J Psychiatry. 2010;167(7):782-91.

12. Locher C, Koechlin H, Zion SR, Werner C, Pine DS, Kirsch I, et al. Efficacy and safety of selective serotonin reuptake inhibitors, serotonin-norepinephrine reuptake inhibitors, and placebo for common psychiatric disorders among children and adolescents: a systematic review and meta-analysis. JAMA Psychiatry. 2017;74(10):1011-20. https://doi.org/10.1001/jamapsychiatry.2017.2432

13. Holtmann M, Bolte S, Poustka F. Suicidality in depressive children and adolescents during treatment with selective serotonin reuptake inhibitors: Review and meta-analysis of the available randomised, placebo controlled trials. Nervenarzt. 2006;77:1332-7.

14. Fegert JM, Kölch M, Zito JM, Glaeske G, Jahnsen K. Antidepressant use in children and adolescents in Germany. J Child Adolesc Psychopharmacol. 2006;16:197-206.

15. Dolle K, Schulte-Körner G. The treatment of depressive disorders in children and adolescents. Dtsch Ärztebl Int. 2013;110(50):854-60.

16. Bogen S, Legenbauer T, Bogen T, Gest S, Jensch T, Schneider S, et al. Morning light therapy for juvenile depression and severe mood dysregulation: study protocol for a randomized controlled trial. Trials. 2013; 14(1):178.

17. Gest S, Legenbauer T, Bogen S, Schulz C, Pniewski B, Holtmann M. Chronotherapeutics: an alternative treatment of juvenile depression. Med Hypotheses. 2014;82(3):346-9.

18. Al-Karawi $D$, Jubair L. Bright light therapy for nonseasonal depression: Meta analysis of clinical trials. J Affect Disord. 2016;198:64-71.

19. Tuunainen A, Kripke D, Endo T. Light therapy for non-seasonal depression. Cochrane Database Syst Rev. 2004;(2):CD004050

20. Popper CW. Mood disorders in youth: exercise, light therapy, and pharmacologic complementary and integrative approaches. Child Adolesc Psychiatr Clin N Am. 2013;22:403-41.

21. Bogen S, Legenbauer T, Gest S, Holtmann M. Lighting the mood of depressed youth: Feasibility and efficacy of a 2 week-placebo controlled bright light treatment for juvenile Inpatients. J Affect Disord. 2016;190:450-6.

22. Legenbauer T, Heiler S, Holtmann M, Fricke-Oerkermann L, Lehmkuhl G. The affective storms of school children during night time: Do affective dysregulated school children show a specific pattern of sleep disturbances? J Neural Transm. 2012:119:989-98.

23. Gest S, Holtmann M, Bogen S, Schulz C, Pniewski B, Legenbauer T. Chronotherapeutic treatments for depression in youth. Eur Child Adolesc Psychiatry. 2016;25(2):151-61.

24. Kirschbaum I, Straub J, Gest S, Holtmann M, Legenbauer T. Short-term effects of wake- and bright light therapy on sleep in depressed youth. Chronobiol Int. 2018;35:101-10. https://doi.org/10.1080/07420528.2017.1388251.

25. Moscovici L, Kotler M. A multistage chronobiologic intervention for the treatment of depression: A pilot study. J Affect Disord. 2009;116:201-7.

26. Viola A, Hubbard J, Comtet H, Hubbard I, Delloye E, Ruppert E, et al. Beneficial effect of morning light after one night of sleep deprivation: Light glasses versus light box administration. Vienna, Austria: Poster. Society for Light Treatment and Biological Rhythms. Annual Meeting; 2014.

27. Kirschbaum-Lesch I, Gest S, Legenbauer T, Holtmann M. Feasibility and efficacy of four weeks of bright light therapy in depressed adolescent inpatients. Z Kinder Jugendpsychiatr Psychother. 2018:46:423-9. https://doi. org/10.1024/1422-4917/a000603.

28. Lam RW, Levitt AJ, Levitan RD, Enns MW, Morehouse R, Michalak EE, et al. The Can-SAD study: a randomized controlled trial of the effectiveness of light therapy and fluoxetine in patients with winter seasonal affective disorder. Am J Psychiatry. 2006;163:805-12.

29. Dilling H, Freyberger HJ. Taschenführer zur Klassifikation psychischer Störungen: ICD-10. Bern: Hogrefe; 1994.

30. Horne JA, Östberg O. A self-assessment questionnaire to determine morningness-eveningness in human circadian rhythms. Chronobiol Int 1976;4:97-110.

31. Wirz-Justice A, Benedetti F, Terman M. Chronotherapeutics for affective disorders: a clinican's manual for light and wake therapy. 2nd ed. Basel: Karger; 2013.

32. Terman JS, Terman M, Lo ES, Cooper TB. Circadian time of morning light administration and therapeutic response in winter depression. Arch Gen Psychiatry Res. 2001:58:69-75.

33. Besier T, Goldbeck L, Keller F. Psychometric properties of the Beck depression inventory-II (BDI-II) among adolescent psychiatric patients. Psychother Psych Med. 2008;58:63-8. 
34. Guy W. Clinical Global Impressions (CGI) Scale. 2000. http://expert-nurse. com/yahoo_site_admin/assets/docs/Clinical-Global-Impressions-Scale_CGI. 162131649.doc. Accessed 25 Oct 2017.

35. Keller F, Grieb J, Ernst M, Sproeber N, Fegert JM, Koelch M. Children's Depression Rating Scale - Revised (CDRS-R): Development of a German version and psychometric properties in a clinical sample. Z Kinder Jugendpsychiatr Psychothery. 2011;39:179-85.

36. Plener PL, Grieb J, Sprober N, Straub J, Schneider A, Keller F, et al. Convergence of children's depression rating scale-revised scores and clinical diagnosis in rating adolescent depressive symptomatology. Ment IIIn. 2012; 4(1):29-31.

37. March JS, Silva S, Vitiello B, TADS Team. The Treatment for Adolescents with Depression Study (TADS): methods and message at 12 weeks. J Am Acad Child Adolesc Psychiatry. 2006;45:1393-403.

38. Goodyer IM, Dubicka B, Wilkinson P, Kelvin R, Roberts C, Byford S, et al. A randomised controlled trial of cognitive behaviour therapy in adolescents with major depression treated by selective serotonin reuptake inhibitors: The ADAPT trial. Health Technol Assess. 2008;12(14) iii-iv:ix-60.

39. Görtelmeyer R. Manual zum SF-A/R und SF-B/R - Schlaffragebogen A und B - Revidierte Fassung. Gießen: Hogrefe; 2011.

40. Tryon WW. The reliability and validity of two ambulatory monitoring actigraphs. Behav Res Methods. 2005;37:492-7.

41. Ravens-Sieberer U, Auquier P, Erhart M, Gosch A, Rajmil L, Bruil J, et al. The KIDSCREEN-27 quality of life measure for children and adolescents: psychometric results from a cross-cultural survey in 13 European countries. Qual Life Res. 2007:16:1347-56.

42. Starfield B, Riley AW, Green BF, Ensminger ME, Ryan SA, Kelleher K, et al. The adolescent child health and illness profile. A population based measure of health. Med Care. 1995;33:553-66.

43. Forrest CB, Tambor E, Riley AW, Ensminger ME, Starfield B. The health profile of incarcerated male youths. Pediatrics. 1997;105:286-91.

44. Woerner W, Becker A, Rothenberger A. Normative data and scale properties of the German parent SDQ. Eur Child Adolesc Psychiatry. 2004;13:ii3-ii10

45. Zimmerman P, Fimm B. A test battery for attentional performance. In: Leclerca M, Zimmerman P, editors. Applied Neuropsychology of Attention Theory, Diagnosis and Rehabilitation. London: Psychology Press; 2002. p. 110-51.

46. Martiny $\mathrm{K}$, Lunde M, Unden M, Dam H, Bech P. High cortisol awakening response is associated with an impairment of the effect of bright light therapy. Acta Psychiatr Scand. 2009;120(3):196-202

47. Oswald, WD, Roth E. Der Zahlen-Verbindungs-Test (ZVT). Ein sprachfreier Intelligenz-Test zur Messung der "kognitiven Leistungsgeschwindigkeit". Handanweisung (2.überarbeitete und erweiterte Auflage). Göttingen: Hogrefe; 1987.

48. Oswald WD. Zahlen-Verbindungs-Test ZVT. (3., überarbeitete und neu normierte Auflage). Göttingen: Hogrefe; 2016.

49. Kasper S, Wehr TA, Bartko JJ, Gaist PA, Rosenthal NE. Epidemiological findings of seasonal changes in mood and behavior. A telephone survey of Montgomery County, Maryland, USA. Arch Gen Psychiatry. 1989:46(9):823-33.

Ready to submit your research? Choose BMC and benefit from:

- fast, convenient online submission

- thorough peer review by experienced researchers in your field

- rapid publication on acceptance

- support for research data, including large and complex data types

- gold Open Access which fosters wider collaboration and increased citations

- maximum visibility for your research: over $100 \mathrm{M}$ website views per year

At BMC, research is always in progress.

Learn more biomedcentral.com/submissions 